\title{
Research on Control Strategy of Hybrid Energy Storage System in Distributed Generation
}

\author{
Chaojiang Guo ${ }^{1, ~ a, ~ S u p i n g ~} \mathrm{Gao}^{2, \mathrm{~b}}$ and Shize $\mathrm{Li}^{3, \mathrm{c}}$ \\ ${ }^{1}$ Harbin Institute of Technology Shenzhen Graduate School, Guangdong, province, Shenzhen \\ ${ }^{2}$ Shenzhen Polytechnic, Guangdong province, Shenzhen \\ ${ }^{3}$ Harbin Institute of Technology Shenzhen Graduate School, Guangdong, province, Shenzhen \\ a1062911600@qq.com, bgaosup@szpt.edu.cn,
}

\begin{abstract}
Keywords: hybrid energy storage system; multi-objective optimization; multiple protections
Abstract. A control strategy which is used in hybrid energy storage system is proposed in this paper. This control strategy has combined low pass filter with multi-objective optimization. Besides, the problems of overcharge, over discharge and limitation of maximum power are considered, which has improved the reliability of the system. The construction of the multi-objective optimization model and the coordination of the multiple protections are introduced in detail. And the advantages of the control strategy are validated by simulation in MATLAB.
\end{abstract}

\section{Introduction}

Storage system plays an important role in the distributed generation. On the one hand, it can smooth the output power of renewable energy sources to improve the power quality; on the other hand, it has the function for peak load shifting, which is favorable to increase operation rate of renewable energy sources. At present, commonly used storage mediums are batteries, super-capacitors and so on. But as the limitation of present energy storage technology, only one kind of storage medium cannot meet the above two needs[1]. A hybrid storage system that combines batteries with super-capacitors can meet the performance requirement and reduce the cost of funds at the same time, so hybrid energy storage system has become research hotspot at home and abroad.

Research has proved that hybrid storage system can make full use of different storage medium, which prolongs the service time of the storage system[2];The use of high pass filter to attribute the power and taking the SOC(State Of Charge) of battery into consideration can prolong the service time of battery[3]; in [4], moving average filtering was used to distribute the power, and the time constant is adjusted base on the SOC of battery, which achieve the reasonable distribution of power between battery and super-capacitor. A control strategy that combines fuzzy control with low pass filtering was proposed in [5]. Low pass filter is used to smooth the power that is offered by battery, the fuzzy control is used to adjust the SOC of battery and super-capacity. But the design of the fuzzy controller is difficult, which need certain of design experience. Neural network algorithm is also applied to control the storage system. On the one hand, it ensures that the battery bear the power of low-frequency, on the other hand, it can minimize energy requirements[6]. However, this control strategy requires a large amount of historical data to train the neural network.

There are already some research results at the control strategy of energy storage system, but few literatures are published aiming at reducing system loss, improving the utilization of super-capacitors and improving the overall performance. In this paper, a multi-objective optimization control strategy is proposed based on the existing research. And the effectiveness of the control strategy is verified by MATLAB / Simulink simulation. 


\section{Structure of Hybrid Energy Storage System in Distributed Generation}

The connection structure of hybrid energy storage system in distributed generation system includes passive connection, semi-active connection and active connection. Active connection is used in this paper, as show in Fig.1. Two kinds of energy storage medium are connected in parallel to the DC bus through their respective bidirectional converters. Compared with the other connection structure, active connection allows achieving both better capacitor utilization and excellent load voltage regulation[7].

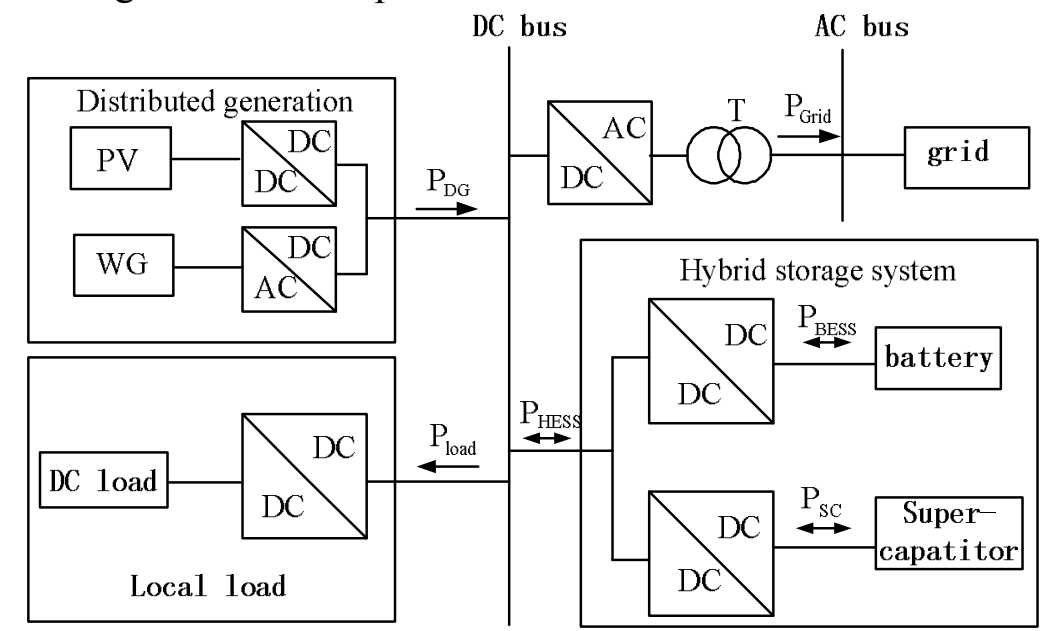

Fig .1 active connection of hybrid storage system in distributed generation

\section{Control strategy of hybrid storage system}

Design of the control strategy. The objective of controlling the hybrid energy storage system is to optimize the overall performance of the system and to extend the service life of the system. This paper design a control strategy which combine low pass filtering with multi-objective optimization, and overcharge ,over discharge, maximum power limitation are take into consideration, as show in Fig. 2.

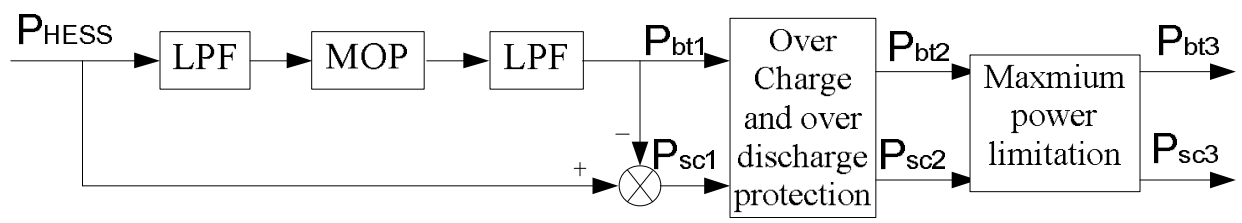

Fig. 2 control strategy diagram

Where $\mathrm{P}_{\text {HESS }}$ is the power that the hybrid power storage system needs to bear; LPF represents the low pass filtering; MOP represents multi-objective optimization; $\mathrm{Pbt}$ is the power that the battery needs to bear; Psc is the power that the super-capacitor need to bear.

Distribution of power. A strategy that combines LPF with MOP is used to distribute the power.LPF ensure that the battery bears the low-frequency power, to reduce the current stress and prolong the service time of battery[8]. The low pass filter is achieved by the Eq.1.

$$
P_{L}=P_{H E S S} \cdot \frac{1}{s T_{f}+1}
$$

Where $T_{f}$ is the time constant of the low pass filter.

After low-pass filter processing, the power $P_{H E S S}$ is divided into low-frequency power and high-frequency power. The high-frequency power is offered by the super-capacitor, the low-frequency power is decomposed again by the multi-objective optimization algorithm. There are two goals for re-decomposing the low-frequency power: 1) Reducing the energy loss of the system; 2) improve the utilization of super-capacitor and overall performance. To achieve the two goals, we should establish the corresponding mathematical model. 


\section{a. Selection of control variables}

In the hybrid energy storage system, the super capacitor and battery terminal voltage change is relatively slow, the voltage can be seen as constant, so power control can be converted to current control. The super-capacitor and the battery are connected in parallel to the DC bus via their bidirectional converter, $P_{H E S S}$ is offered by the battery and super-capacitor, so the distribution of power can be determined by determining the current output of one converter. In this paper, the battery current is chosen as the control variable, the output current of the super-capacitor can be expressed as Eq.2.

$$
i_{s c}=\frac{P_{H E S S}-i_{b t} V_{b t}}{V_{s c}}
$$

\section{b. Establishment of Loss Optimization Model}

There we set the energy loss of the system as $P_{\text {loss }}$, it is shown in Eq.3 :

$$
P_{\text {loss }}=P_{b t . l o s s}+P_{\text {scl.loss }}+P_{\text {conl.loss }}+P_{\text {con2.loss }} .
$$

Where $P_{b t . l o s s}$ is the battery internal resistance loss; $P_{\text {sc.loss }}$ is the super-capacitor internal resistance loss; $P_{\text {con1.loss }}$ and $P_{\text {con2.loss }}$ are the loss of the converters. In (3): $P_{b t . l o s s}=i_{b t}^{2} R_{b t}, P_{s c . l o s s}=i_{s c}^{2} R_{s c} \quad, \quad P_{\text {con1.loss }}=V_{b u s} f_{s}\left|i_{s c}\right|\left(t_{r}+t_{f}\right)+i_{s c}^{2}\left(R_{L 1}+R_{s 2} D_{2}+R_{s 1} D_{1}\right)$, $P_{\text {con2.loss }}=V_{b u s} f_{s}\left|i_{b t}\right|\left(t_{r}+t_{f}\right)+i_{b t}^{2}\left(R_{L 2}+R_{s 4} D_{4}+R_{s 3} D_{3}\right) . R_{b t}$ and $R_{s c}$ are the internal resistance of battery and super-capacitor; $V_{b u s}$ is the DC voltage; $f_{s}$ is the switching frequency; $t_{r}$ and $\mathrm{t}_{\mathrm{f}}$ is the rise time and fall time of the switch; $R_{L 1}$ and $R_{L 2}$ is Parasitic resistance of inductors; $R_{s 1} \sim R_{s 4}$ is the switching on-resistance; is the duty of switch.

The energy loss optimization model is established base on the Eq.4.

$$
\left(P_{1}\right)\left\{\begin{array}{l}
\min \quad U\left(i_{b t}\right)=\int_{0}^{T}\left|\frac{P_{l o s s}\left(i_{b t}\right)}{P_{H E S S}}\right| d t \\
\text { s.t. } \quad \frac{P_{H E S S}-V_{s c} i_{s c . \text { max }}}{V_{b t}} \leq i_{b t} \leq i_{b t \text { max }}
\end{array} .\right.
$$

Where $i_{b t \text { max }}$ and $i_{s c \text { max }}$ is the maximum output current of battery and super-capacitor.

c. Optimization Model of Super Capacitor Utilization and Overall Performance

Optimization Model of Super Capacitor Utilization and Overall Performance can be converted to the control of SOC of super-capacitor. The optimization model is established as Eq.5.

$$
\left(P_{2}\right)\left\{\begin{array}{ll}
\min \quad & S\left(i_{b t}\right)=\int_{0}^{T}\left|\frac{S O C_{s c}\left(i_{b t}\right)-S O C_{s c}^{*}}{S O C_{s c \text { max }}-S O C_{s c \text { min }}}\right| d t \\
\text { s.t. } & \frac{P_{H E S S}-V_{s c} i_{s c . \text { max }}}{V_{b t}} \leq i_{b t} \leq i_{b t \text { max }}
\end{array} .\right.
$$


Where the $S O C_{s c}^{*}$ is the expected SOC, when $P_{H E S S}>0$, hybrid power storage system discharge, $S O C_{s c}^{*}$ is $S O C_{s c . l o w}$, on the contrary, $S O C_{s c}^{*}$ is $S O C_{s c . h i g h}$.

The optimization model can improve the utilization of super-capacitor and the performance of the whole storage system.

d. integration of the multi-objection

It is difficult to realize the above-mentioned optimization targets at the same time, so we should consider all objections for different control purpose. Here we introduce the weighting factor, and the integral model is established as Eq.6.

$$
\left(P_{\text {final }}\right)\left\{\begin{array}{ll}
\min & F\left(i_{b t}\right)=\lambda U\left(i_{b t}\right)+(1-\lambda) S\left(i_{b t}\right) \\
\text { s.t. } & \frac{P_{H E S S}-V_{s c} i_{s c \text { max }}}{V_{b t}} \leq i_{b t} \leq i_{b t \text { max }}
\end{array} .\right.
$$

Where $\lambda$ is the weighting factor.

This integral model can simplify the optimization model and the solving process.

Protection of over charge and discharge. In the process of charging and discharging, overcharge and over discharge is inevitable, which will seriously affect the life of battery and super capacitor. To avoid the situation, we take certain measure. First, we divide the SOC of battery and super-capacitor into five areas, forbidden charging area, alert charging area, normal area, alert discharging area and forbidden discharging area, as show in Fig.3.

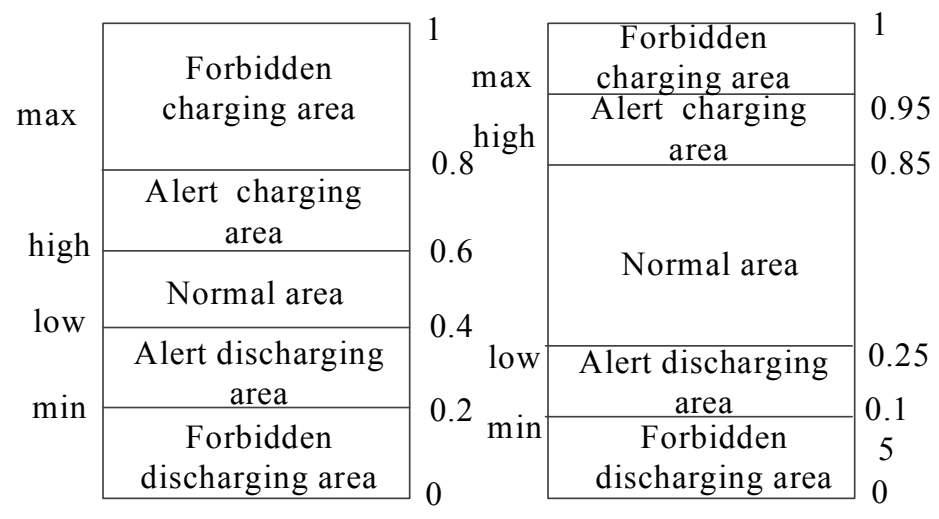

a)division of battery b)division of super-capacitor

Fig.3 division of storage's SOC

Then, we make corresponding treatment to the power for different state of the storage system. When the energy storage system is discharging, if it is in the discharging alert area, the discharge power needs to be treated as Eq.7; when the energy storage system is charging, if it is in the charging alert area, the charging power needs to be treated as Eq.8.

$$
\begin{aligned}
P_{d i s}^{x} & =P_{d i s}^{x} \cdot \max \left\{0, \frac{S O C_{x}-S O C_{\min }}{S O C_{l o w}-S O C_{\min }}\right\} . \\
P_{c h}^{x} & =P_{c h}^{x} \cdot \max \left\{0, \frac{S O C_{\max }-S O C_{x}}{S O C_{\max }-S O C_{\text {high }}}\right\} .
\end{aligned}
$$


From (7) and (8) we can find that if the power was dealt with (7) or (8), it will be smaller. So the two energy storage devices need to coordinate with each other to complete the power instruction better. The processing of coordination is shown in the Fig.4.

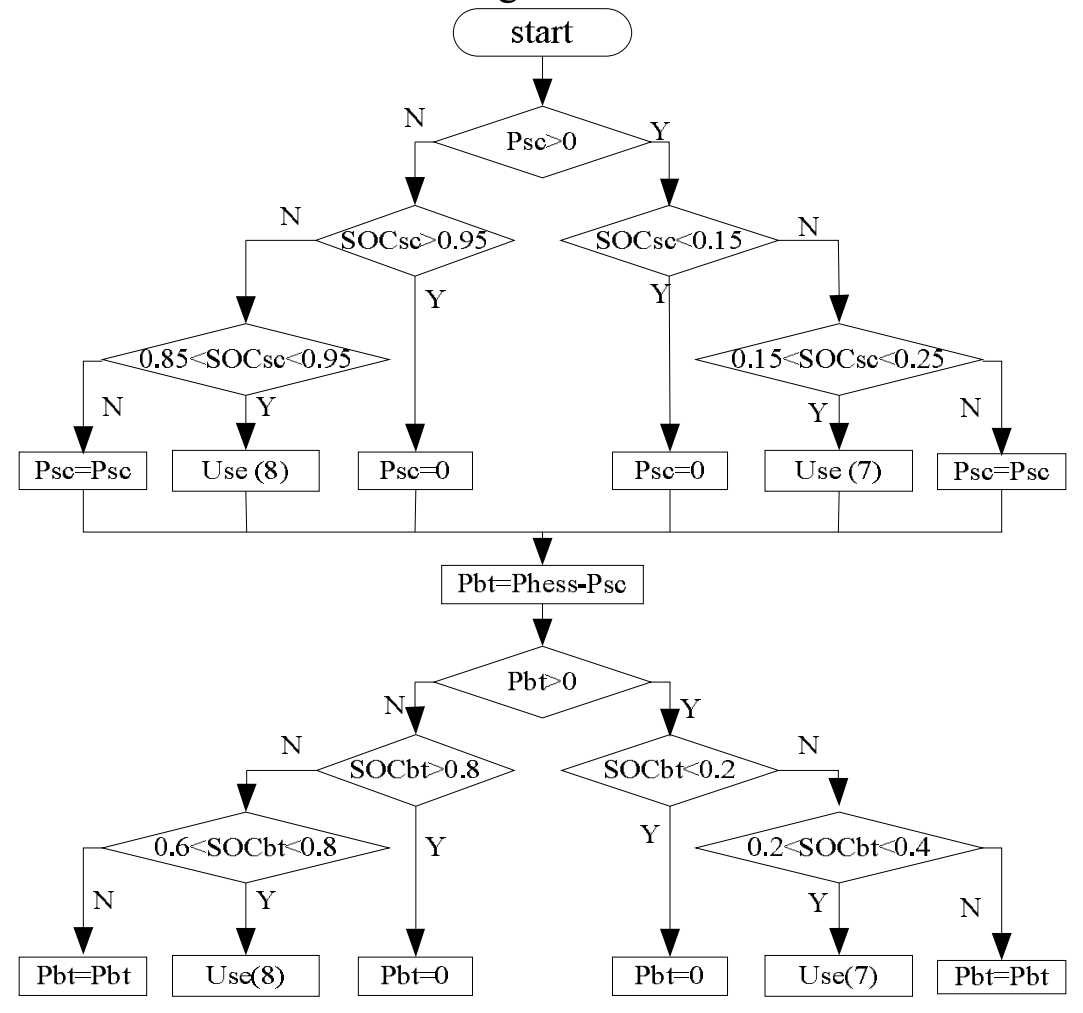

Fig. 4 the processing of coordination

In the processing of charging or discharging, super-capacitor is more prone to be at the state of overcharge or over discharge, so in the processing of coordination, the power of super-capacitor is dealt firstly.

Limitation of maximum power. In the charging and discharging process, the two energy storage devices have their own maximum input and output power limit. When the power instruction exceeds the limitation, the excess portion should be borne by the other storage device reasonably. For example, when the power instruction of super-capacitor exceeds the limitation, we deal with the power instruction as Eq.9 and Eq.10.

$$
\begin{aligned}
& P_{s c 3}=P_{s c . \text { max }} . \\
& P_{b t 3}=P_{b t 2}+P_{s c 2}-P_{s c \text { max }} .
\end{aligned}
$$

We should ensure that the power instruction of battery doesn't exceed the power limitation. For other situation, we handle it with the same method to ensure the safe of the storage system.

\section{Simulation and analysis}

To verify the effectiveness of the control strategy, the system model of the distributed generation is built with the structure in fig. 1 in MATLAB/Simlink. And we do some simplifications, the DC load is replaced by an adjustable resistor, renewable source and grid is replaced by a controlled current source. The model of battery is the general model, the model of super-capacitor is the First order RC model. 
The power instruction $P_{\text {HESS }}$ is replace by a random fluctuation power with the amplitude of $2 \mathrm{KW}$. The main parameters of the simulation are shown in Table 1.

Table 1 Main parameter of power distribution simulation

\begin{tabular}{cccc}
\hline parameter & value & parameter & value \\
\hline$S O C_{b t 0}$ & 0.5 & $R_{s 1} \sim R_{s 4}$ & $0.1 \Omega$ \\
\hline$S O C_{s c 0}$ & 0.5 & $Q_{b t}$ & $10 \mathrm{Ah}$ \\
\hline$T_{f}$ & $30 \mathrm{~s}$ & $Q_{s c}$ & $20 \mathrm{~F}$ \\
\hline$f_{s}$ & $10 \mathrm{kHz}$ & $i_{b t \cdot \max }$ & $30 \mathrm{~A}$ \\
\hline$t_{r}$ & $0.5 \mathrm{us}$ & $i_{s c \cdot \max }$ & $50 \mathrm{~A}$ \\
\hline$t_{f}$ & $0.5 \mathrm{us}$ & $\lambda$ & 0.5 \\
\hline$R_{\mathrm{L} 1} R_{\mathrm{L} 2}$ & $1 \Omega$ & &
\end{tabular}

The targets of the control strategy are as follows:

1)complete the system power instruction;

2)the battery bear low-frequency power, super-capacitors bear the high-frequency power;

3)improve the utilization of super capacitor;

4)reduce the overall loss of the system;

The simulation results of the control strategy are show in Fig.5 Fig.8.

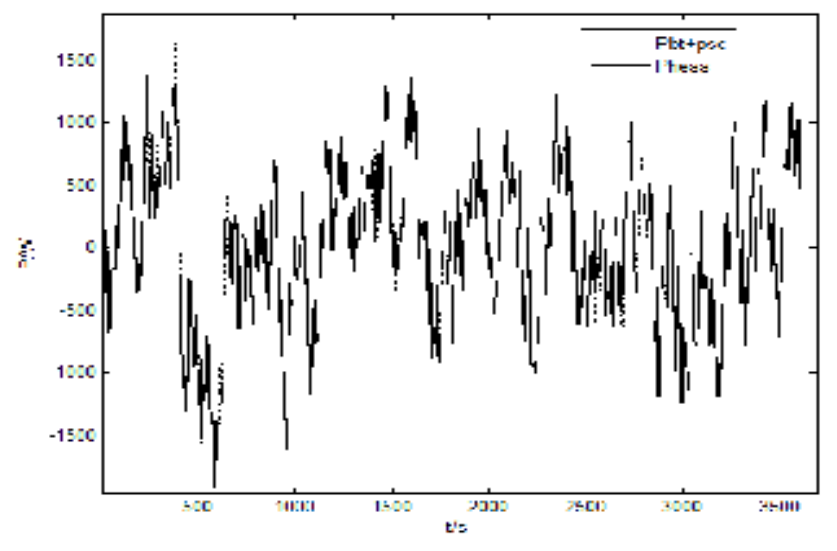

Fig. 5 Completion of HESS power instruction

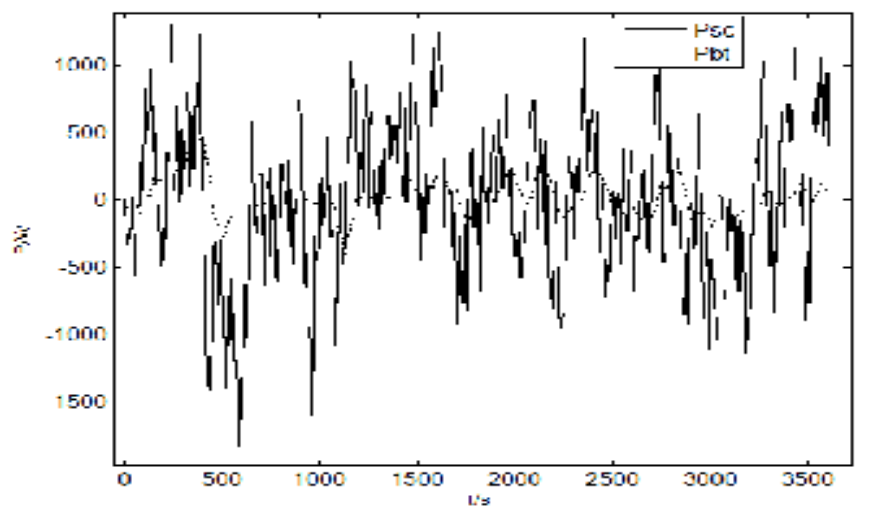

Fig.6 waveform of Pbt and Psc 


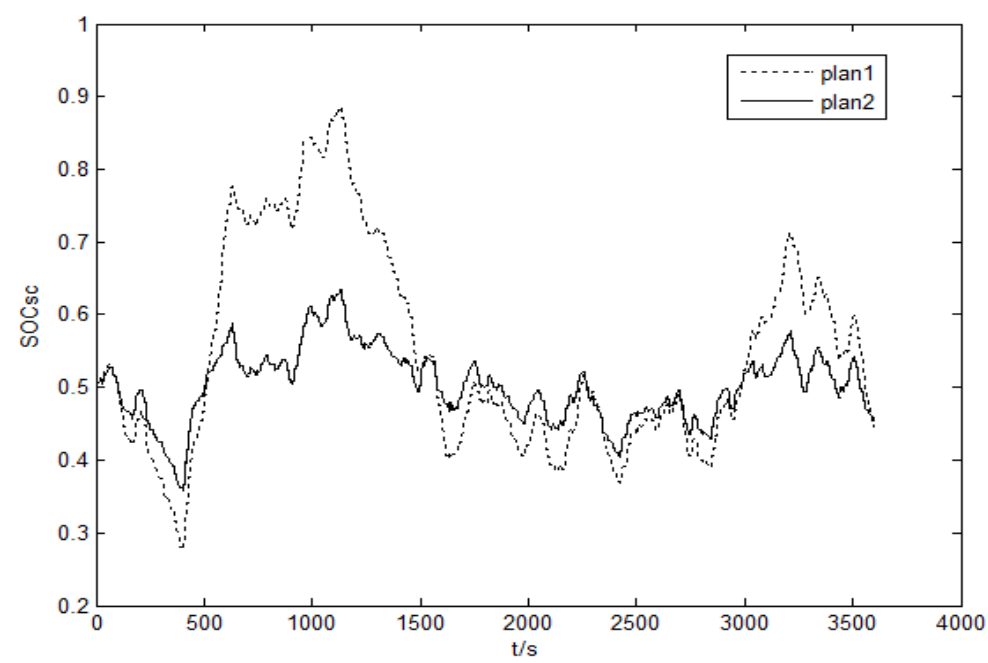

Fig.7 compare of SC's SOC

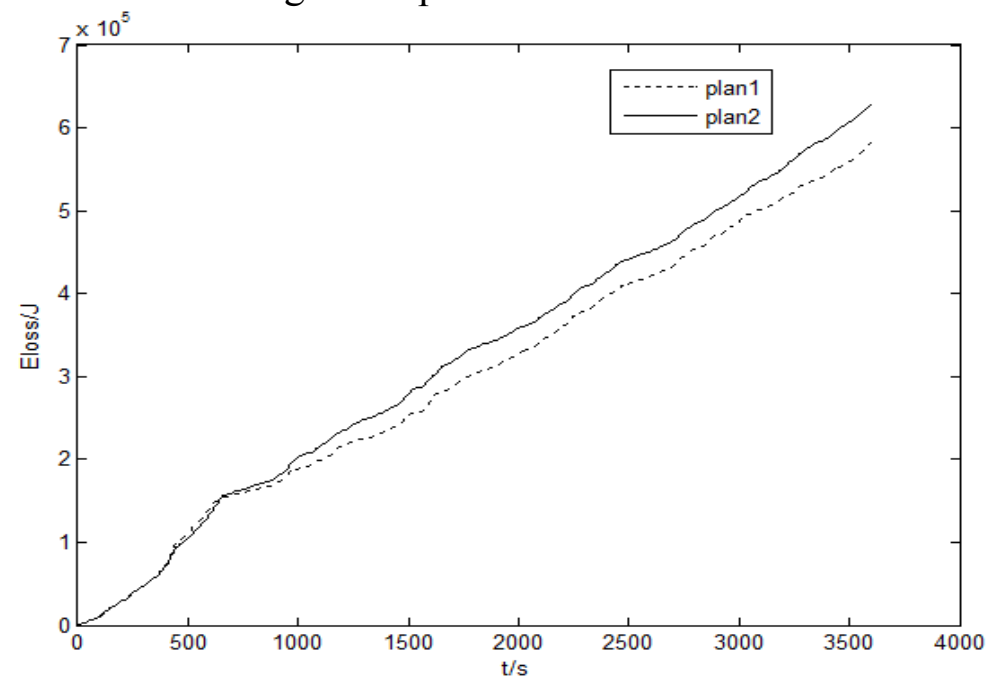

Fig. 8 compare of energy loss

The Fig. 5 shows the completion of the power instruction, and the Fig. 6 shows the distributed results of the control strategy. From the results we can conclude that the control strategy can complete the power instruction well, and can ensure that super-capacitor bears most of the high-frequency power, the battery bears the low-frequency power, which will reduce the current stress of battery and prolong the service time.

To highlight the advantages of the proposed control strategy (plan1), we make a comparison between plan1 and traditional control strategy (plan2). The results of comparison are shown in Fig.7 and Fig.8. Fig.7 shows that the utilization of plan1 is bigger than plan2. Fig.8 shows that the energy loss of plan1 is smaller than plan2.

From these simulation results we can conclude that the proposed control strategy can achieve the control targets well.

\section{Conclusions}

In this paper, a control strategy of hybrid energy storage system is proposed, which combines low-pass filter with multi-objective optimization. It can ensure that the battery bears the low-frequency power and the super-capacitor works in healthy state, which prolong the service time of the storage system. At the same time, the proposed control strategy can improve the utilization of super-capacitor and reduce the energy loss of the system. The overall performance of the energy storage system has been improved. The following work of this paper is to verify the strategy in experiment. 


\section{Acknowledgements}

This work was financially supported by Shenzhen Municipal Bureau of Science and Technology Commission project (JCYJ20140718172055170).

\section{References}

[1] Reza Hemmati, Hedayat Saboori. Emergence of hybrid energy storage systems in renewable energyand transport applications - A review [J]. Renewable and Sustainable Energy Reviews, 2016, 65(1): 11-23.

[2] Dougal R A, Liu S, White R E. Power and life extension of battery-ultracapacitor hybrids[J]. IEEE Trans on Component stand Packaging technologies, 2002, 25(1): 120-130.

[3] Nishad Mendis, Kashem M. Management of Battery-Supercapacitor Hybrid Energy Storage and Synchronous Condenser for Isolated Operation of PMSG Based Variable-Speed Wind Turbine Generating Systems [J]. IEEE Transactions on smart grid, 2014 5(2): 944-952.

[4] Zhang Guoju, Tang Xisheng, Qi Zhiping. Design of Hybrid Energy Storage System for Intermittent Power Supply [J]. Automation of Electric Power Systems, 2011，35(20): 24-28.

[5] Yanzhu Ye, Pawan Garg, Ratnesh Sharma. Development and Demonstration of Power Management of Hybrid Energy Storage for PV Integration [J]. IEEE PES Innovative Smart Grid Technologies Europe. 2013(4): 1-5.

[6] Jorge Moreno, Micah E. Ortúzar, Juan W. Dixon. Energy-Management System for a Hybrid Electric Vehicle, Using Ultracapacitors and Neural Networks [J]. IEEE Transactions on industrial electronics,2012 53(2): 614-623.

[7] Jian Cao, Ali Emadi. A new Battery/ultraCapacity Hybrid Energy Storage System for Electric, Hybrid, and Plug-in Hybrid Electric Vehicles[J].IEEE Transaction on Power Electronics, 2012 27(1):122-132.

[8] Ziyou Song, Heath Hofmann, Jianqiu Li, Jun Hou, Xuebing Han, Minggao Ouyang. Energy management strategies comparison for electric vehicles with hybrid energy storage system[J].Applied Energy, 134(2014):321-331. 